\title{
The dynamic interplay between a cell fate determinant and a lysozyme homolog drives the asymmetric division cycle of Caulobacter crescentus
}

\author{
Sunish Kumar Radhakrishnan, ${ }^{1}$ Martin Thanbichler, ${ }^{2}$ and Patrick H. Viollier ${ }^{1,3}$ \\ ${ }^{1}$ Department of Molecular Biology and Microbiology, School of Medicine, Case Western Reserve University, Cleveland, \\ Ohio 44106, USA; ${ }^{2}$ Max Planck Institute for Terrestrial Microbiology, D-35043 Marburg, Germany
}

Caulobacter crescentus divides asymmetrically into a swarmer cell and a stalked cell, a process that is governed by the imbalance in phosphorylated levels of the DivK cell fate determinant in the two cellular compartments. The asymmetric polar localization of the DivJ kinase results in its specific inheritance in the stalked daughter cell where it phosphorylates DivK. The mechanism for the polar positioning of DivJ is poorly understood. SpmX, an uncharacterized lysozyme homolog, is shown here to control DivJ localization and activation. In the absence of $\mathrm{SpmX}$, DivJ is delocalized and dysfunctional, resulting in developmental defects caused by an insufficiency in phospho-DivK. While SpmX stimulates DivK phosphorylation in the stalked cell, unphosphorylated DivK in the swarmer cell activates an intricate transcriptional cascade that leads to the production of the $\operatorname{spmX}$ message. This event primes the swarmer cell for the impending transition into a stalked cell, a transition that is sparked by the abrupt accumulation and localization of SpmX to the future stalked cell pole. Localized SpmX then recruits and stimulates DivJ, and the resulting phospho-DivK implements the stalked cell fate. The dynamic interplay between SpmX and DivK is at the heart of the molecular circuitry that sustains the Caulobacter developmental cycle.

[Keywords: Asymmetric cell division; cell fate determinant; polar protein localization; muramidase; kinase] Supplemental material is available at http://www.genesdev.org.

Received August 6, 2007; revised version accepted November 21, 2007.

Asymmetric division is fundamental to differentiation in eukaryotes and prokaryotes. A signature of this process is the generation of an imbalance in the distribution and/or activity of cell fate determinants in daughter cells, endowing them with distinctive features (Horvitz and Herskowitz 1992; Shapiro and Losick 1997). For example, in the asymmetric division of Drosophila neuroblasts, a protein kinase (aPKC) within the Par complex is polarized to the apical membrane. This asymmetric disposition of aPKC, along with its cell cycle activation, governs the timely phosphorylation of a cell fate determinant (Lgl) in one of the two daughter cells (Wirtz-Peitz and Knoblich 2006). A remarkably similar principle operates at the heart of the developmental program that instructs stem cell-like division in the crescent-shaped bacterium Caulobacter crescentus (Fig. 1). A protein kinase (DivJ) that is asymmetrically localized and activated during the cell cycle phosphorylates a cell fate de-

${ }^{3}$ Corresponding author.

E-MAIL patrick.viollier@case.edu; FAX (216) 368-1055.

Article is online at http://www.genesdev.org/cgi/doi/10.1101/gad.1601808. terminant (DivK) specifically in one of the two daughter cells (Skerker and Laub 2004). Here we describe an uncharacterized muramidase homolog (SpmX) that acts as spatiotemporal regulator of DivJ and DivK.

The Caulobacter predivisional cell features a cylindrical extension of the cell envelope, the stalk, at the old cell pole and a rotating flagellum along with the pili synthetic apparatus at the new pole (Fig. 1A). A stalked daughter cell that replicates its chromosome and a swarmer daughter cell that is maintained in a (G1-like) nonreplicative state are the products of each cell division. DivJ is localized to the stalked pole in the predivisional cell (Fig. 1A) and is inherited by the stalked daughter cell where it phosphorylates DivK, an essential single domain response regulator. The $\mathrm{PleC}$ phosphatase is localized to the flagellated pole and, directly and possibly also indirectly, antagonizes Divj to maintain DivK P levels low in the swarmer cell compartment (Fig. 1A; Matroule et al. 2004; Skerker and Laub 2004).

DivK implements the fate of the two daughter cells through CtrA (Fig. 1B; Wu et al. 1998), a DNA-binding response regulator that is stimulated by phosphorylation to interact with its target sites (CtrA boxes) (Quon et al. 
1996). The stability and phosphorylation of CtrA are cell cycle-regulated (Fig. 1A; Domian et al. 1997), and DivK controls both these events. In the stalked cell, DivK P triggers the removal of phosphorylated CtrA (CtrA P) (Hung and Shapiro 2002; Biondi et al. 2006a; Iniesta et al. 2006). In the swarmer cell, CtrA P represses DNA replication by binding to five target sites within the chromosomal origin of replication (Cori) (Quon et al. 1998). In addition, $\mathrm{Ctr} A \sim \mathrm{P}$ regulates transcription at promoters of developmental genes, including pilA, which encodes the structural subunit of the pilus filament that is extruded from the flagellated pole of swarmer cells (Skerker and Shapiro 2000; Laub et al. 2002). A hallmark of the Caulobacter division cycle is that the swarmer cell morphs into a replicative stalked cell in response to an unknown cell cycle cue. In doing so, a stalk elaborates from the pole previously vacated by ejecting the flagellum and retracting the pili. Coincident with these mor-

Figure 1. Graphical representation of the cell fate regulators in Caulobacter and the genetic circuit they constitute. (A) Subcellular localization of DivJ, DivK, PleC, and the localization factors PodJ and SpmX during the Caulobacter asymmetric division cycle: SpmX (red dot) localizes to the pole previously occupied by PleC (green dot), which eventually morphs into the stalked pole. SpmX recruits DivJ (blue dot), initiating the phosphorylation and localization of DivK (yellow), eventually resulting in bipolar DivK P (yellow dot) in predivisional cells. Predivisional cells also display PleC at the flagellated pole. PleC has been recruited previously to that site by PodJ (purple dot), which localizes to that pole in late stalked cells (Viollier et al. 2002b; Hinz et al. 2003). Coincident with the compartmentalization of the late predivisional cell, localized DivK P and diffuse (dephosphorylated) DivK are present in the stalked (ST) cell chamber and the swarmer (SW) cell chamber, respectively. Pili and the flagellum are indicated by the thin lines (black) and the thick wavy line, respectively. The circular dashed arrow denotes a rotating flagellum. The graded black bar indicates the time during the cell cycle that $\mathrm{CtrA} \sim \mathrm{P}$ is present. In late predivisional cells, CtrA P accumulates in the swarmer compartment and is eliminated from the stalked cell compartment. $(B)$ In the swarmer cell compartment (SW, red), PleC reduces DivK P levels by direct dephosphorylation of DivK P (rectangular arrow, event 1) (Matroule et al. 2004), and possibly indirectly by inhibiting the DivJ kinase (Sommer and Newton 1991; Wheeler and Shapiro 1999) by the production of an inhibitor or modulator of DivJ activity. High levels of DivK P inhibit CtrA P-mediated transcriptional activation of $t a c A$ and pilA by a mechanism that is poorly understood (Biondi et al. 2006a). The tacA translation product along with $\sigma^{54}$-containing RNA polymerase $\left(E \sigma^{54}\right)$ catalyzes transcription of $\operatorname{spmX}$ and $s t a R$, the gene for a transcriptional regulator of stalk biogenesis (Biondi et al. 2006b). At the swarmer-to-stalked cell transition, SpmX accumulates to localize and activate DivJ (event 2), thereby producing a surge in DivK P that signals the removal of CtrA P by a complex phosphosignaling cascade (Biondi et al. 2006a; Iniesta et al. 2006). This event, coupled with the disappearance of TacA at the swarmer-to-stalked cell transition (see Fig. 4A), leads to the shut-down of SpmX transcription in stalked cells (ST, blue). The dotted lines indicate transcriptional regulation; bold lines indicate the regulation at the level of protein localization or activity. The black dashed arrow indicates the new connections of the circuit uncovered herein. phological changes, CtrA phosphorylation subsides and the protein is degraded. This relieves repression of Cori and DNA replication commences in the nascent stalked cell (Domian et al. 1997), events bearing functional resemblance to the eukaryotic G1-S transition.

The inactivation of $\mathrm{CtrA} \sim \mathrm{P}$ is signaled by a surge in DivK $\sim$, caused by the abrupt localization and activation of DivJ at the pole previously occupied by PleC (Fig. 1A; Wheeler and Shapiro 1999; Hung and Shapiro 2002). Mutations in $\operatorname{div} J$ and $\operatorname{div} K$ result in elevated CtrA activity and aberrant cell division, and a mutation in $\operatorname{ctr} A$ can overcome the requirement of DivK for viability (Wu et al. 1998; Hung and Shapiro 2002; Pierce et al. 2006). In the absence of PleC, CtrA P levels are reduced, DivK $\sim \mathrm{P}$ levels are dramatically elevated, and DivJ is delocalized yet still able to efficiently phosphorylate DivK (Wheeler and Shapiro 1999; Biondi et al. 2006a). The finding that DivK P levels are lower, but not higher, in the pleC divJ double mutant compared with the divJ single mutant (Wheeler and Shapiro 1999) begs the question of whether in addition to controlling Divj localization, PleC plays a role in preventing precocious activation of DivJ (Fig. 1B). $\mathrm{PleC}$ is know to promote stalk elongation, flagellum rotation, and pilA transcription (Ohta et al. 1992; Wang et al. 1993; Viollier and Shapiro 2003).

Since the PleC-DivJ-DivK phosphosignaling cascade

\section{A}

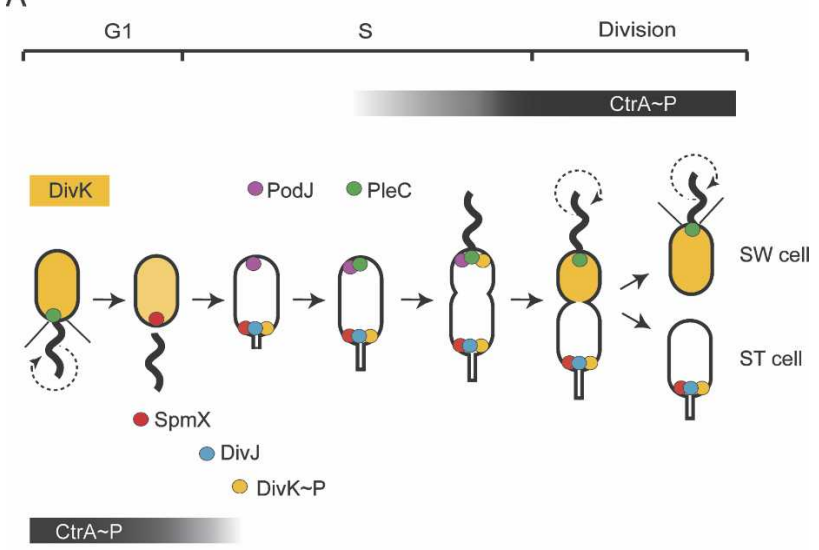

B

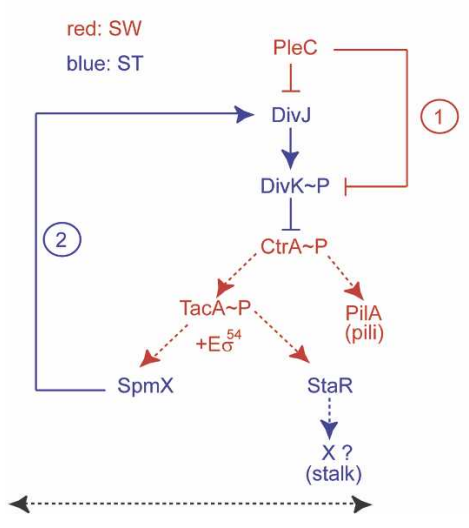


impinges on motility, we explored if additional components of this pathway can be identified in a screen for motility mutants. Using such a screen (S. Pritchard, D. Matteson, E. Huitema, S.K. Radhakrishnan, and P.H. Viollier, in prep.), we here unveil the uncharacterized muramidase homolog SpmX as a PleC-dependent protein that promotes localization and activation of DivJ, and that therefore indirectly stimulates the surge of DivK P at the G1-S transition. We also found that DivK feeds back on SpmX by way of a transcriptional cascade that relies on the sequential action of CtrA and the recently characterized transcriptional activator TacA that directs stalk elongation at the G1-S interface (Biondi et al. 2006b). We propose that SpmX and DivK represent critical nodes of the regulatory circuitry that sustains the Caulobacter asymmetric division cycle.

\section{Results}

In the absence of the SpmX muramidase homolog, cell division is perturbed

To identify additional regulators of DivK, we screened our comprehensive library of transposon motility mutants for developmental defects by light and transmission electron microscopy (TEM). Strains NS190 and NS349 frequently have bipolar stalks and are perturbed in cell division, a phenotype resembling that of a strain with a cold-sensitive mutation in $\operatorname{divK}$ (divKcs) grown under permissive conditions (Fig. 2C; Supplemental Table S1). Moreover, they were found to be flagellated, but to swim poorly. NS190 and NS349 harbor a Himar1 and an EZ-Tn5 insertion, respectively, in the uncharac- terized CC2173 gene (henceforth referred to as $\operatorname{spmX}$ for stalked pole muramidase homolog) (Fig. 2A). The spmX gene encodes a polytopic membrane protein of 431 residues with two membrane-spanning domains (residues 366-388 and 398-420) near the C terminus and a lysozyme/muramidase-like domain (cd00737; residues 7-143) near the $\mathrm{N}$ terminus (Fig. 2A). In lysozyme, this domain hydrolyzes the $\beta$-1,4-glycosidic bond between $N$ acetylmuramic acid and $N$-acetylglucosamine of the peptidoglycan cell wall (Holtje 1996). An in-frame deletion in $\operatorname{spmX}(\Delta \operatorname{spmX})($ Fig. 2A) gave rise to a motility and division defect as did the spmX transposon insertions in strains NS190 and NS349 (Fig. 2B; data not shown). These results, along with complementation experiments (see Supplemental Fig. S1), indicate that the observed defects are due to the loss of SpmX.

SpmX mutant cells have a strong propensity to elongate into either smooth (unconstricted) filamentous cells or cells with multiple constrictions that are often found at aberrant positions (Fig. 2D,F, G; Supplemental Fig. S2). These aberrant divisions likely underlie the occurrence of minicell-like particles that are occasionally observed in spmX cultures (Supplemental Fig. S2B). The motility defect of spmX mutants (Fig. 2B) could be due to inefficient flagellar rotation or the presence of ectopic flagella that are often observed at the division plane, where pili are also located. The abnormal location of these organelles at the division plane can be reconciled with the observed cell separation defect of $\Delta s p m X$ mutants. If sister cells remain associated with one another and the developmental program proceeds unhindered, this unseparated "post-divisional" cell will grow a stalk at each end. Similarly, pili and flagella will eventually emerge from
Figure 2. A motility mutant screen identifies $\operatorname{spmX}$, an uncharacterized gene encoding a muramidase homo$\log$ required for proper cell division. $(A)$ Domain organization of SpmX showing the predicted transmembrane domains (brown), the muramidase domain (yellow), the position of the Himar1 (white triangle), and the EZ-Tn5 (black triangle) insertion in strains NS349 and NS190, respectively. The line below the domain architecture shows the deleted coding region of the $\Delta \operatorname{spmX}$ strain. (B) Motility assay of NA1000 (wild type), NS190, NS349, $\Delta$ spmX, $\Delta$ pleC, and $\Delta$ divJ strains. Overnight cultures $(2.5 \mu \mathrm{L})$ were placed on PYE swarm $(0.3 \%)$ agar plates and incubated for $60 \mathrm{~h}$ at $30^{\circ} \mathrm{C}$. Motility defects can be seen as swarms with a compact appearance, whereas those from wild type are diffuse and enlarged. $(C-G)$ Transmission electron micrographs of wild-type $(E), \Delta \operatorname{spm} X(D, F, G)$, and $\operatorname{divKcs}(C)$ cells grown at $30^{\circ} \mathrm{C}$. Pili (visualized indirectly by staining with pilus-specific bacteriophage $\Phi \mathrm{CbK}$ ) and flagella are indicated by white and black arrows, respectively. Arrowheads indicate bipolar stalks (black) or abnormal constrictions (white). Bars, $200 \mathrm{~nm}$.
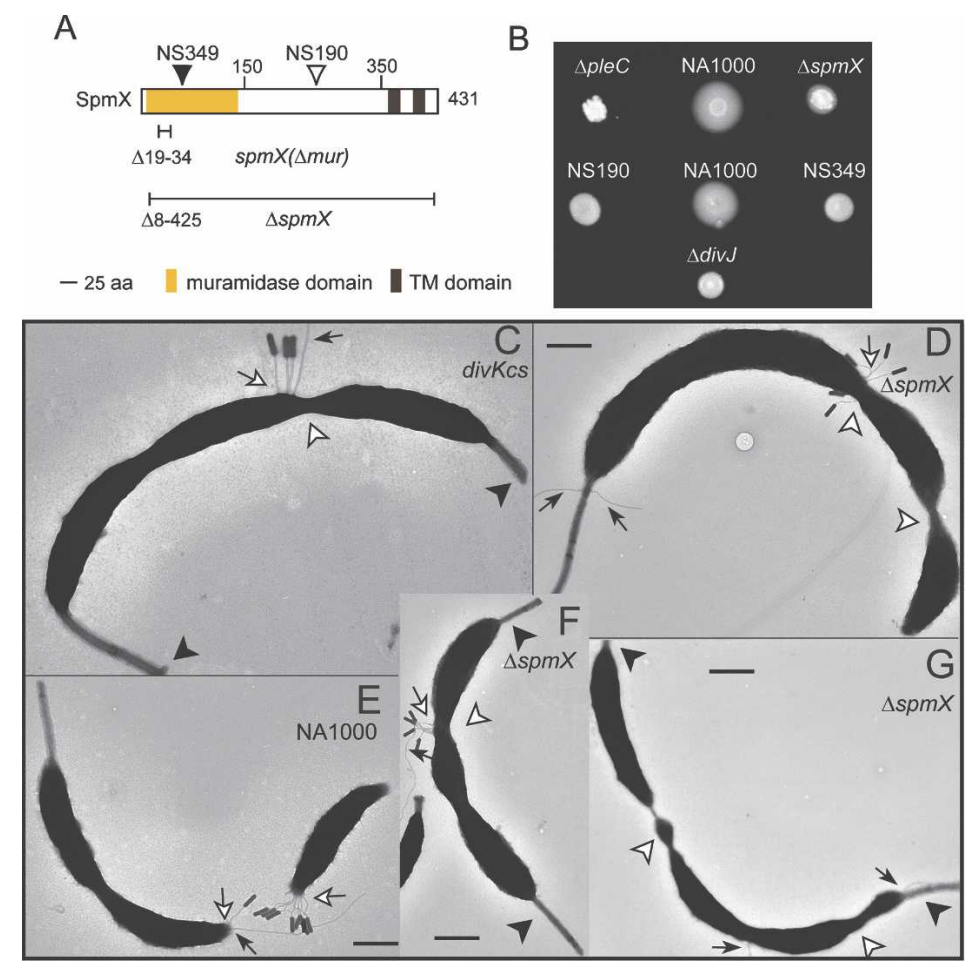
constrictions where functional, albeit physically associated, poles are located. Based on the results shown below, we propose that the phenotypes of $\operatorname{spmX}$ mutants are primarily due to improper regulation of DivK and DivJ.

\section{SpmX controls the localization and activity of DivJ and DivK}

The similarity of divKcs and $\Delta$ spmX mutants prompted us to investigate if SpmX impinges on DivK activity. Phosphorylation of DivK affects its activity as well as its ability to localize to the cell poles (Lam et al. 2003). A low-copy plasmid harboring divK-gfp under the control of the native $\operatorname{div} K$ promoter was transformed into wildtype and $s p m X^{-}$cells to determine if DivK is localized and phosphorylated in the absence of SpmX. Live-cell fluorescence microscopy revealed that, in contrast to the intense polar foci of DivK-GFP in wild-type cells, only diffuse fluorescence was observed in cells lacking SpmX (Fig. 3A; Supplemental Fig. S3A). Immunoblotting using a DivK-specific antiserum demonstrated that the absence of SpmX had no impact on DivK or DivK-GFP steady-state levels (Supplemental Fig. S3B). Thus, SpmX is required for DivK localization.

To test if SpmX affects DivK phosphorylation, we determined DivK $\sim \mathrm{P}$ levels in $\Delta s p m X$ and $\Delta$ divj cells relative to wild-type cells (Fig. 3C). Consistent with previous results (Wheeler and Shapiro 1999), DivK P levels are severely reduced in $\Delta$ divJ cells, because they lack DivJ, the principal kinase for DivK (Figs. 1B, 3C). Similarly, in $\Delta$ spmX cells, DivK P levels were $72 \%$ lower than in wild-type cells. This result is consistent with the idea that inefficient phosphorylation of DivK underlies its inability to localize to the poles of $\Delta$ spmX cells. Moreover, this result raised the possibility that, akin to divJ mutations, the perturbation in cytokinesis of $\Delta s p m X$ cells is a consequence of low DivK P. If so, then a pleC mutation, which causes a dramatic increase in cellular DivK P levels (Wheeler and Shapiro 1999), might ameliorate the cytokinetic abnormalities by raising DivK P levels. Microscopic examination revealed that $\Delta$ spmX $\Delta p l e C$ double-mutant cells localize DivK-GFP to the cell poles and have lost the characteristic cell division phenotype of spmX single mutants (Fig. 3A,B). Instead, the cells had acquired the hallmarks of $\Delta p l e C$ cells (stalkless and pililess) (Fig. 3A; data not shown). The relative levels of DivK $\sim \mathrm{P}$ were elevated in $\Delta \operatorname{spmX} \Delta$ pleC double-mutant cells and were comparable with those observed in the $\Delta$ pleC single mutant (Fig. 3C). To confirm that this effect was caused by the loss of activity rather than the physical absence of PleC, we deleted $\operatorname{spmX}$ in the pleC(H610A) strain, in which the pleC gene bears a point mutation encoding catalytically inactive PleC (PleCH610A) (Viollier et al. 2002a). The phenotype of the resulting $\Delta$ spmX pleC $(H 610 A)$ double mutant was indistinguishable from that of the $\Delta s p m X \Delta$ ple $C$ mutant (data not shown), confirming that the loss PleC activity can ameliorate the $\Delta s p m X$ phenotype.

Histidine kinases like DivJ first undergo a transient
A

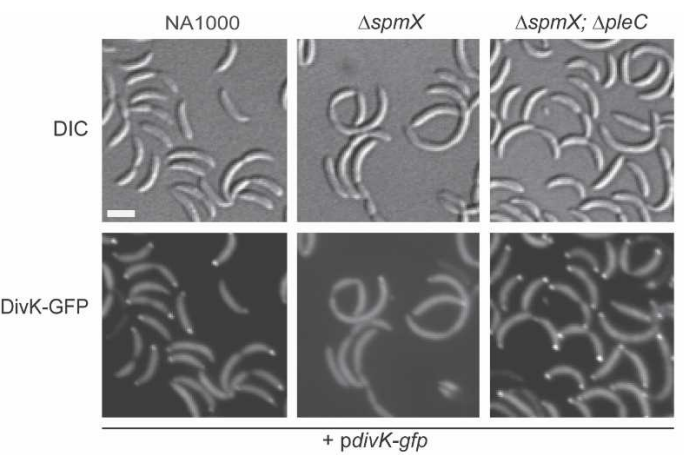

B
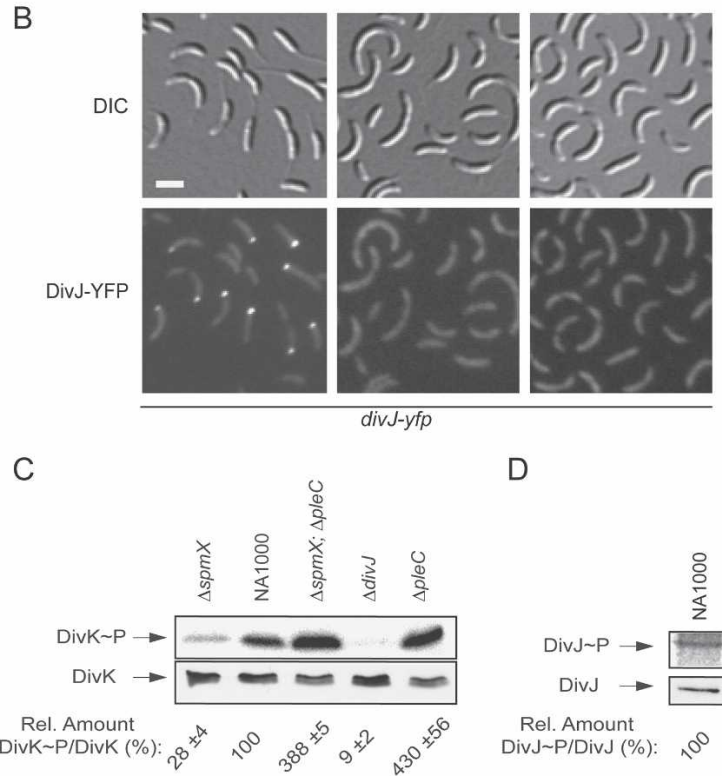

$\mathrm{D}$

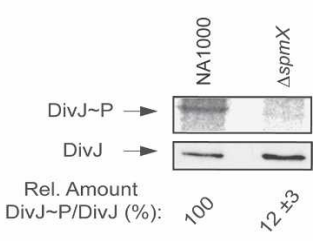

Figure 3. Localization and in vivo phosphorylation of DivK and DivJ are impaired in the $\Delta \operatorname{spm} X$ mutant. $(A, B)$ Differential interference contrast (DIC) and fluorescence micrographs of NA1000, $\Delta s p m X$, and $\Delta$ spmX $\Delta p l e C$ cells expressing either DivK-GFP expressed from a low-copy plasmid (pdivK-gfp) under the control of the native $\operatorname{divK}$ promoter $(A)$ or DivJ-YFP from the endogenous divJ locus $(B) .(C)$ Determination of relative DivK P/DivK levels in NA1000, $\Delta$ spmX, $\Delta$ pleC $\Delta s p m X, \Delta p l e C$, and $\Delta$ divj cells. $(D)$ Measurement of relative Divj P/DivJ levels in NA1000 and $\Delta \operatorname{spm} X$ cells. Bars, $\sim 2 \mu \mathrm{m}$.

trans-autophosphorylation event at a conserved histidine residue before the phosphate is passed on to a response regulator, such as DivK (Stock et al. 1990). Because the results above suggested that DivJ kinase activity is compromised in $\Delta \operatorname{spmX}$ cells, we measured the relative levels of autophosphorylated DivJ (DivJ P) in wild-type and $\Delta \operatorname{spmX}$ mutant cells. DivJ P levels were dramatically reduced $(88 \%)$ (Fig. $3 \mathrm{D})$ in $\Delta$ spmX cells compared with wild-type cells. This result, along with the requirement of SpmX for efficient DivK phosphorylation, indicates that DivJ activity is compromised in the $\Delta$ spmX mutant. Epistasis experiments provided additional evidence that SpmX lies in a genetic pathway with DivJ and DivK. The phenotype of $\Delta s p m X \Delta$ divJ doublemutant cells is indistinguishable from that of the $\Delta d i v J$ single mutant (data not shown), showing that the effects 
of $\Delta$ divJ and $\Delta s p m X$ deletions, each of which compromise DivK phosphorylation on their own, are not additive.

Because DivJ is localized to the stalked pole, we tested the idea that SpmX controls both the polar positioning and activation of DivJ. Accordingly, we constructed $\Delta$ spmX strains in which the divJ gene was replaced with a variant encoding either DivJ-YFP (Fig. 3B) or DivJ-GFP (Supplemental Fig. S3E) under control of the divJ promoter. Analysis of these strains by fluorescence microscopy along with a $s p m X^{+}$isogenic parent revealed only diffuse fluorescence in $\Delta s p m X$ cells or in $\Delta s p m X \Delta p l e C$ cells (Fig. 3B). Immunoblot analysis using antibodies to DivJ showed DivJ-YFP or DivJ-GFP steady-state levels were maintained in the $\Delta \operatorname{spm} X$ mutant (Supplemental Fig. S3D,F). Based on these results we conclude that SpmX is required (1) to direct DivJ to the stalked pole, (2) to stimulate autophosphorylation of DivJ, and, therefore, (3) to catalyze efficient phosphotransfer from DivJ to DivK. Because DivJ kinase activity is dispensable for DivJ localization to the stalked pole (Lam et al. 2003), we surmise that the effect of SpmX on DivJ localization is not a secondary consequence of a primary effect on kinase activity. Instead, we propose that the principal role of SpmX is to direct DivJ to the stalked pole and to activate it upon its recruitment to the cell pole.

\section{SpmX localizes to the nascent stalked pole} during the G1-S transition

To explore if SpmX is localized to the same subcellular site as DivJ, we visualized SpmX by live-cell fluorescence microscopy in cells producing SpmX translationally fused to the $\mathrm{C}$ terminus or the $\mathrm{N}$ terminus of the red fluorescent protein mCherry. Strains bearing these modified alleles in place of wild-type $\operatorname{spmX}$ appear phenotypically normal as determined by differential interference contrast (DIC) microscopy, indicating that these SpmX chimeras are functional. Live-cell fluorescence microscopy of these strains revealed a bright fluorescent focus of SpmX-mCherry or mCherry-SpmX at the stalked pole (Fig. 4B). To corroborate this localization data, we also visualized SpmX in fixed cells by indirect immunofluorescence microscopy (IFM) using a polyclonal antibody raised against SpmX (Fig. 4C). Unipolar foci derived from SpmX were observed in wild-type cells, but not in $\Delta$ spmX mutant cells. Because stalk integrity is compromised by the cell permeabilization procedure used during IFM, we were unable to unambiguously assign the location of the SpmX signal to the stalked pole. To resolve this ambiguity, SpmX was immunolocalized in cells expressing the stalked pole marker DivJ-GFP and was found to colocalize with DivJ-GFP (Fig. 4C). While cells that had polar DivJ-GFP always contained overlapping SpmX signals, the converse was not true: Occasionally cells with polar SpmX were observed in which DivJ is not localized. This observation, along with the finding that SpmX is required for DivJ localization, suggested that polar localization of SpmX precedes that of DivJ. This prompted us to construct a divJ-yfp spmX-mCherry strain to colocalize SpmX and DivJ in live cells. Consistent with the IFM results, SpmX-mCherry and DivJ-YFP colocalize in cells of an unsynchronized culture (Fig. 4D). Time-lapse fluorescence microscopy of synchronized swarmer cells was used to determine the temporal sequence of localization of SpmX and DivJ. Initially, neither SpmX nor DivJ was localized. Subsequently, polar SpmX-mCherry foci appeared, followed by the emergence of DivJ-YFP foci and subsequently the stalk (Fig. $4 \mathrm{E}$ ) at the same pole. To determine whether or not SpmX is present in swarmer cells, we probed blots containing extracts prepared from wild-type cells at different stages of the cell cycle with the anti-SpmX antibody. As shown in Figure 4A, the SpmX protein is at first barely detectable in swarmer cells $(t=0 \mathrm{~min})$, but accumulates sharply during the transition into stalked cells $(t=20$ min) and is present henceforth. An identical pattern of abundance was observed in $s p m X$-mCherry cells (Supplemental Fig. S4). Thus, the accumulation and localization of SpmX is an early event during the G1-S transition that is required for and precedes the recruitment of DivJ to the nascent stalked pole.

Prompted by the findings above, we explored whether SpmX and DivJ reside in the same complex at the stalked pole using coimmunoprecipitation experiments. A monoclonal antibody to GFP was used to precipitate DivJ-YFP from membrane-solubilized extracts of the divJ-yfp strain. Immunoblotting showed that SpmX was present in this sample, but not in the sample in which NA1000 solubilized extracts were processed in an identical manner (Fig. 4F). In a reciprocal experiment, DivJYFP could be coimmunoprecipitated with the antiSpmX antibodies from extracts of the divJ-yfp strain, but not from those of the $\Delta s p m X$; divJ-yfp strain (Fig. $4 \mathrm{H}$ ). In contrast, two other proteins that are detected by the anti-GFP antibody (Fig. 4G) and the polarly localized McpA chemoreceptor (Supplemental Fig. S5) were not present in the precipitates from both extracts. These experiments indicate that DivJ and SpmX reside in the same protein complex, providing a mechanistic basis for the notion that DivJ is attracted to the stalked cell pole via the prior localization of SpmX. DivJ is predicted to have six TM domains and to lack major segments that protrude into the periplasm (Sommer and Newton 1991), suggesting that this complex is membrane-anchored.

\section{Role of the muramidase domain in SpmX and DivJ localization}

To explore if the muramidase domain is required for SpmX function and/or localization, we engineered alleles encoding $\operatorname{SpmX}(1-150)$, SpmX(1-350), and

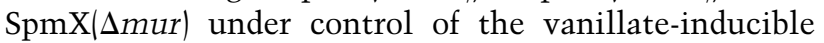
promoter, $\mathrm{P}_{\text {van }}$ on a low-copy plasmid (Thanbichler et al. 2007). SpmX(1-150) just encompasses the muramidase domain, SpmX(1-350) lacks the two TM segments, and $\operatorname{SpmX}(\Delta$ mur $)$ is deleted for the active site residues from E19-T34 (see Fig. 2A). Compared with wild-type SpmX, none of the mutants was able to support DivJ 
A
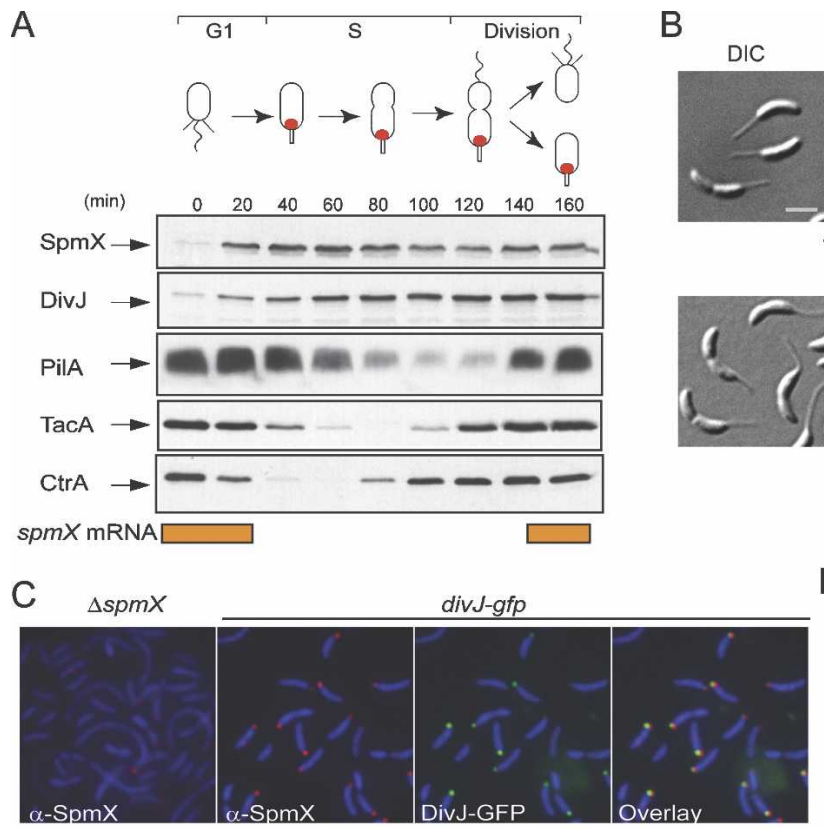

D
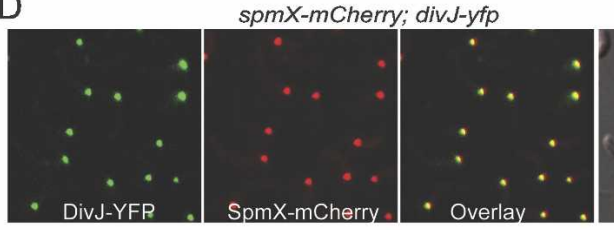

E (nis)

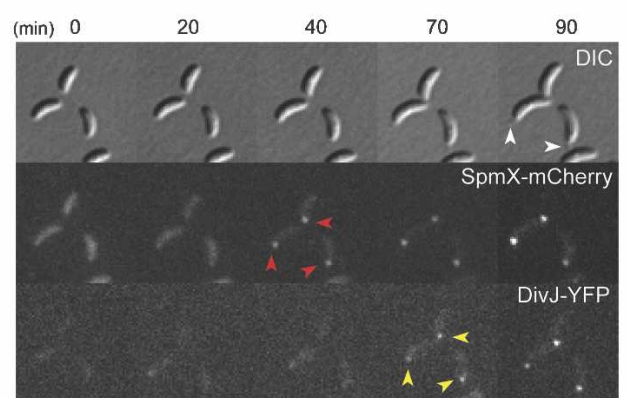

F

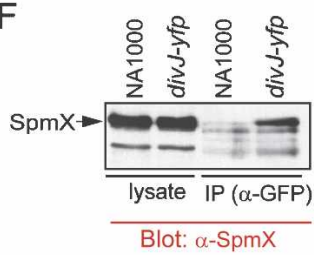

B
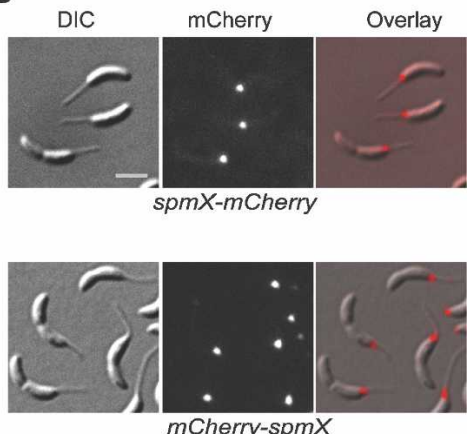

G

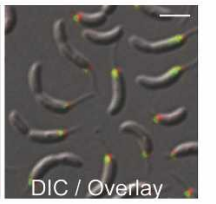

$\mathrm{H}$
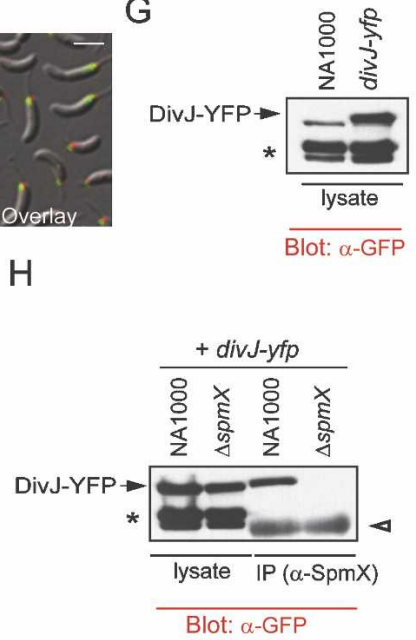

Figure 4. Colocalization of SpmX and DivJ. (A) Immunoblots showing cellular levels of SpmX, DivJ, PilA, TacA, and CtrA during various stages of the wildtype (NA1000) cell cycle. The orange bars indicate the time when spmX mRNA is present during the cell cycle as determined previously (Laub et al. 2000; McGrath et al. 2007). (B) Polar localization of SpmX-mCherry and mCherry-SpmX, expressed from the endogenous chromosomal locus in place of wild-type SpmX in strains MT237 and MT272, respectively. (C) Immunofluorescence micrographs using anti-SpmX antiserum in LS3200 (divJ$g f p$ ) cells. SpmX-derived signals (red) colocalize (yellow) with DivJ-GFP signals (green). Cells were visualized by staining in $1 \mu \mathrm{g} / \mathrm{mL}$ DAPI $\left(4^{\prime}\right.$,6-diamidino-2-phenylindole). No SpmX-derived foci were seen at the poles in $\Delta$ spmX cells. $(D)$ DivJYFP (green) and mCherry-SpmX (red) colocalize (yellow) to the stalked pole of NR3601 (spmX-mCherry; divJ-yfp) cells in which the modified alleles replaced the native spmX and divJ genes. (E) Timelapse fluorescence microscopy of purified NR3601 swarmer cells growing on a cushion of $1 \% \mathrm{M} 2 \mathrm{G}$ agarose on a microscope slide, showing that SpmX-mCherry (red arrowheads) localizes to the pole first, followed by that of DivJ-YFP (yellow arrowheads), and finally the stalk (white arrow heads) elongates from the same pole. Bars, $\sim 2 \mu \mathrm{m}$. (F) Immunoprecipitation (IP) analysis of membrane-solubilized extracts of the divI-yfp and NA1000 strain with a monoclonal antibody to GFP ( $\alpha$-GFP). Precipitated samples were analyzed by immunoblotting (Blot) using specific polyclonal antibodies to $\operatorname{SpmX}(\alpha-\operatorname{SpmX}) \cdot(G)$ Immunoblot analysis of extracts from NA1000 and divJ-yfp to detect DivJ-YFP using a monoclonal antibody to GFP. The asterisk $\left(^{\star}\right)$ marks two nonspecific signals reacting with the anti-GFP antibody. $(H)$ Immunoprecipitation experiments using membrane solubilized extracts of the divJ-yfp and $\Delta s p m X$; divJ-yfp strains and polyclonal antibodies to SpmX. DivJ-YFP was detected by immunoblotting using a monoclonal antibody to GFP. Note that the two nonspecific signals $\left({ }^{*}\right)$ do not coprecipitate. The white arrowhead marks the position of the signals derived from the IgG heavy chains.

localization or wild-type morphology when expressed in the $\Delta \operatorname{spmX}$; divf-yfp strain (Fig. 5A). Immunoblotting showed that this deficiency was not due to a failure to produce SpmX(1-350) or SpmX(Dmur) (Fig. 5B). To investigate if these mutants were localized to the stalked pole, we created translational fusions to the $\mathrm{N}$ terminus of mCherry and localized the fusion proteins in NA1000 (Fig. 5C) and the $\Delta \operatorname{spm} X$ mutant (data not shown). The localization results were similar in both backgrounds and revealed that the muramidase domain contains information sufficient to direct mCherry to the stalked pole. In contrast, $\operatorname{SpmX}(\Delta m u r)$-mCherry was expressed (Fig. 5D) but delocalized, suggesting that an intact muramidase active center is critical for localization. Be- cause SpmX(1-350) (Fig. 5E), but not the cytoplasmic protein CtrA (Fig. 5F), was released into the supernatant upon spheroblasting, we surmise that $\operatorname{SpmX}(1-350)$ is found in the periplasmic compartment and that the inability to recruit Divj to the stalked pole is not due to its absence from the periplasm. The fact that SpmX(1-150) retains the ability to localize to the stalked pole suggests that it is also exported to the periplasmic space (Fig. 5B).

Together, our results support a model in which an intact muramidase active center serves to target SpmX to the stalked pole. The subsequent recruitment of DivJ into a complex at the stalked pole by SpmX is likely mediated via membrane interactions requiring the SpmX transmembrane domains. We propose that in this com- 
Figure 5. Role of the muramidase domain in the localizing of SpmX and DivJ to the stalked pole. $(A)$ Fluorescence and DIC micrographs of $\Delta s p m X$; divJyfp cells expressing $\mathrm{SpmX}$ derivatives from a vanillate-inducible promoter $\left(\mathrm{P}_{\text {van }}\right)$ on low-copy plasmid pRVMCS-5 (Thanbichler et al. 2007) after growth in PYE supplemented with tetracycline $(1 \mu \mathrm{g} / \mathrm{mL})$ and induced with vanillate $(50 \mathrm{nM})$ for $2 \mathrm{~h}$. (B) Immunoblot analysis of SpmX and CtrA steady-state levels present in equal amounts of cellular extracts from strains in $A$. Note that the $\operatorname{SpmX}(1-150)$ derivative is not efficiently detected by the anti-SpmX antibody because a recombinant SpmX derivative from residues $120-351$ was used as immunogen. (C) Localization of SpmX-mCherry derivatives expressed from pRVMCS-5 in NA1000 cells grown as in $A$. Note that as a result of ectopic expression from $\mathrm{P}_{\text {van }}$ SpmX-mCherry can often be seen to localize to the pole opposite the stalk. (D) Immunoblot experiments to determine SpmX-mCherry steady-state levels present in cell extracts from strains in $C$. Equal amounts of cell extracts were loaded in each lane. Similar localization results and abundance patterns as in $C$ and $D$, respectively, were observed when the SpmX-mCherry derivatives were expressed in a $\Delta s p m X$ background (data not shown). (E) NA1000 cells expressing the SpmX-mCherry or SpmX(1-350)-mCherry (as in $D$ ) were converted to spheroblasts (SB) and gently centrifuged. The cell (SB Cells) and supernatant (SB Sup.) fraction, along with a control lysate of untreated cells (UT Lysate), were examined for the presence of SpmX-mCherry or CtrA by immunoblotting. The presence of SpmX $(1-$

A

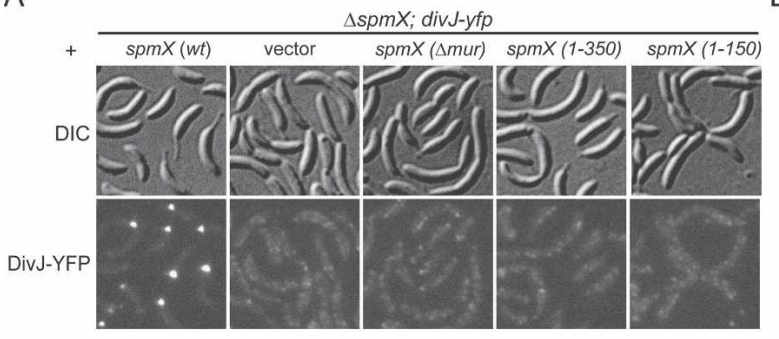

B

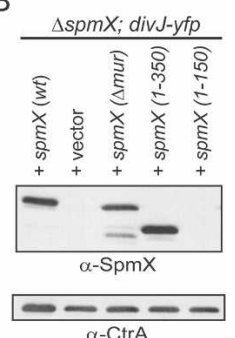

C

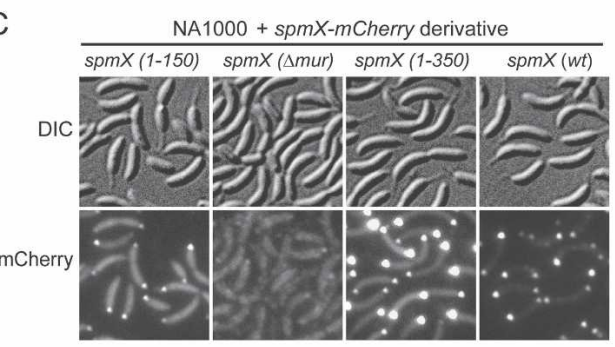

D

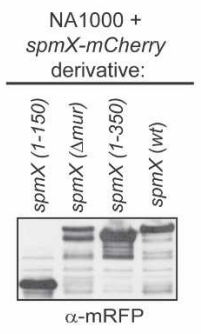

E

NA1000 +

spmX-mCherry derivative:

$\operatorname{spmX} \underline{\operatorname{spm} X(1-350)}$

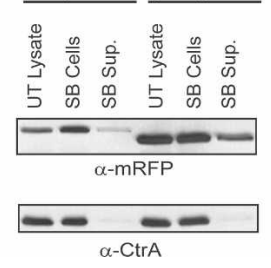

350)-mCherry in the spheroblast supernatant indicates that it is exported into the periplasm in untreated cells. Note that spheroblasting is incomplete, explaining why a substantial amount of SpmX(1-350)-mCherry remains associated with cells. Similar experiments with SpmX(1-150)-mCherry were inconclusive because spheroblasts were fragile, undergoing lysis during centrifugation (data not shown). (F) The immunoblot shown in $E$ was reprobed for the presence of a cytoplasmic protein (CtrA) as a control for cell lysis. In $B, D, E$, or $F$, SpmX-mCherry derivatives and CtrA were detected using polyclonal antibodies to $\operatorname{SpmX}(\alpha-\operatorname{SpmX}), \mathrm{CtrA}(\alpha-\mathrm{CtrA})$, and $\mathrm{mRFP}(\alpha-\mathrm{mRFP})$. Note that the anti-mRFP antibody reacts with mCherry.

plex, activated Divj will then catalyze efficient phosphotransfer to DivK.

\section{PleC regulates Divj localization via transcriptional control of spmX}

It is known that $\mathrm{PleC}$ is required for polar localization of DivJ (Wheeler and Shapiro 1999), but whether or not this is due to a direct or an indirect mechanism is unclear. Based on two lines of evidence we speculated that the latter is true and that SpmX is the centerpiece of this pathway. First, SpmX is necessary to localize DivJ (Fig. 3), and second, mRNA profiling experiments indicated that the abundance of the spmX message is positively dependent on PleC (Chen et al. 2006). The prospect that PleC impinges on the spatiotemporal regulation of DivJ by controlling $\operatorname{spm} X$ transcription prompted us to investigate the underlying mechanism in detail.

First, we confirmed that SpmX levels are dramatically reduced in $\Delta p l e C$ cells by immunoblot analysis of extracts from wild-type and $\Delta p l e C$ mutant cells (Fig. 6A), and that this effect requires the phosphatase activity of
PleC (Supplemental Fig. S6). Next, we tested if $\operatorname{spmX}$ transcription is impaired in $\Delta p l e C$ mutant cells. To this end, we engineered a transcriptional fusion of the $\operatorname{spmX}$ promoter $\left(\mathrm{P}_{\text {spmX }}\right)$ to a promoterless lac $Z$ gene on a lowcopy plasmid and introduced the $\mathrm{P}_{s p m X}-1 a c Z$ reporter construct into the wild-type and $\Delta p l e C$ mutant strain. Quantification of lacZ-encoded $\beta$-galactosidase activity revealed that the $\mathrm{P}_{\text {spmX }}-1 a c Z$ reporter is only $10 \%$ as active in $\Delta p l e C$ mutant cells as in wild-type cells (Fig. 6C). Thus, PleC is required to activate transcription of $\operatorname{spmX}$ as well as that of the pilA pilin gene. Incidentally, the messages of $\operatorname{spmX}$ and pilA exhibit nearly identical abundance patterns during the cell cycle: Both are highly abundant in swarmer cells, are down-regulated in stalked cells, and reaccumulate coincident with the compartmentalization of the predivisional cell (Laub et al. 2000; McGrath et al. 2007). This pattern is no surprise for the pilA message since the abundance profile of the PilA protein essentially reflects that of the message (Fig. 4A). However, for the $\operatorname{spmX}$ mRNA this pattern is unexpected, since the SpmX translation product accumulates during the G1-S transition when $\operatorname{spmX}$ transcript 
A
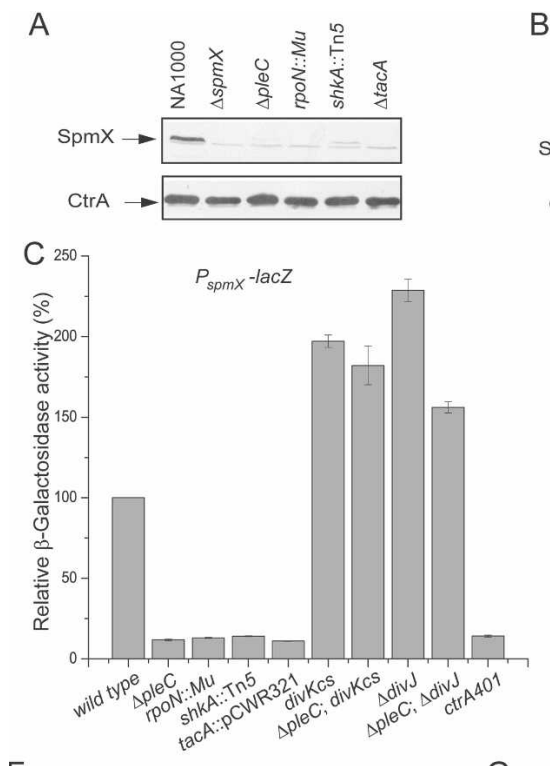

F

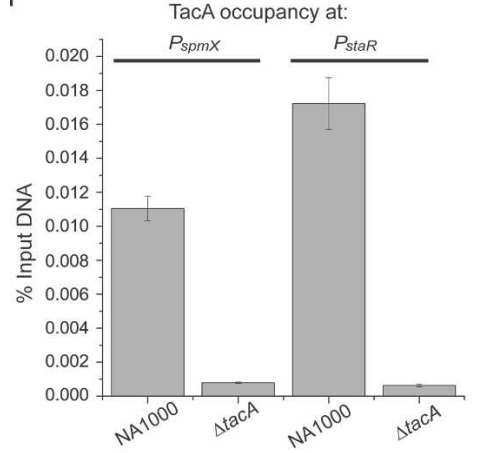

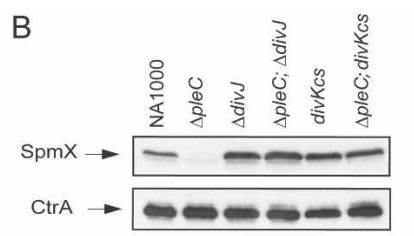

$\mathrm{D}$

$$
\begin{aligned}
& \sigma^{54} \text { consensus: }
\end{aligned}
$$

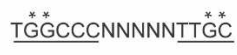

$$
\begin{aligned}
& P_{\text {spmx }} \text { : } \\
& \underline{T^{*} \stackrel{*}{G} C G \underline{C} C G A C C} \underline{T T \stackrel{*}{*}}
\end{aligned}
$$

E

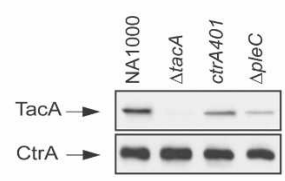

G

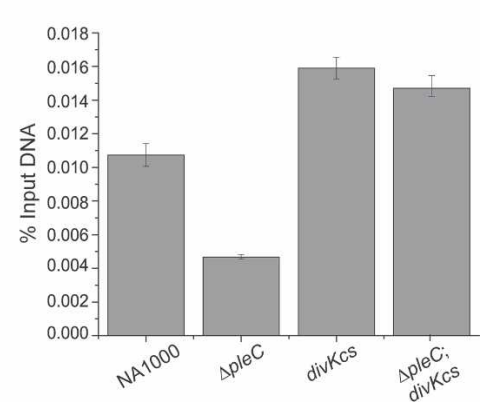

Figure 6. Transcriptional regulation of $\operatorname{spmX}$. (A) Immunoblots showing SpmX steady-state levels in wildtype (NA1000), $\Delta$ pleC, rpoN::Tn5, shkA::Tn5, and $\Delta$ tac $A$ mutants. (B) Immunoblots showing that $\Delta$ divJ and divKcs mutations restore SpmX production to $\Delta$ ple $C$ mutant cells. (C) $\beta$-Galactosidase assays using the $\mathrm{P}_{\text {spmX-lac }}$ reporter plasmid to determine spmX promoter activity in NA1000 and various mutants. Temperature-sensitive mutants were grown at the permissive temperature. $(D)$ Comparison of the spmX promoter sequence with the $\mathrm{E} \sigma^{54}$ consensus sequence. Asterisks $\left({ }^{*}\right)$ indicate nucleotides required for $\mathrm{E \sigma}^{54}$ binding. Underlined are the -24 and -12 consensus sequences for $E \sigma^{54}$ promoters. (E) Immunoblot showing TacA steady-state levels in NA1000, $\Delta$ tacA, ctrA401, and $\triangle p l e C$ mutants. CtrA was used as a loading control for the immunoblots in $A, B$, and $E$. (F) Measurement of TacA occupancy at the spmX and staR promoters in vivo using qChIP analysis. (G) qChIP experiments showing the reduction in CtrA occupancy at the tacA promoter in $\Delta$ ple $C$ mutant cells, and an increase in the divKcs single mutant and the $\Delta$ pleC divKcs double mutant relative to NA1000. levels begin to decline. This suggests that SpmX accumulation is regulated at the post-transcriptional level. Since the spmX and pilA messages are both dependent on $\mathrm{PleC}$, and since $\mathrm{CtrA} \sim \mathrm{P}$ levels are markedly reduced in the pleC mutant (Biondi et al. 2006a), the possibility existed that CtrA P also directly activates transcription from the spmX promoter, along with that of pilA (Fig. $1 B)$. In support of this, $\mathrm{P}_{\text {spmX }}-1 a c Z$ was only $10 \%$ as active in cells with a temperature-sensitive loss-of-function mutation in $\operatorname{ctr} A$ (ctrA401) as in wild-type cells (Fig. 6C). Moreover, SpmX was barely detectable by immunoblot analysis in extracts from ctrA401 cells (Fig. 7B). To determine whether CtrA binds to $\mathrm{P}_{\text {spmx }}$ in vivo, we conducted quantitative chromatin immunoprecipitation (qChIP) experiments using a polyclonal antibody to CtrA. Control qChIP experiments showed that CtrA interacts efficiently with the promoters of pilA and fliL (Supplemental Fig. S7A), both promoters that CtrA P binds to in vitro (Wu et al. 1998; Skerker and Shapiro 2000). However, the $s p m X$ promoter was at least an order of magnitude less abundant in these immunoprecipitated fragments than the fliL or pilA promoters. In fact, precipitation of $\operatorname{spm} X$ promoter DNA was in the same range as the background signal obtained when pilA promoter DNA was immunoprecipitated with antibodies to the CpaE structural protein of the pilus assembly ma- chinery (data not shown), a protein that is not known to bind promoters (Skerker and Shapiro 2000). Based on these results, we surmise that regulation of $\mathrm{P}_{\text {spmX }}$ by CtrA is indirect (Fig. 1B).

\section{A spmX transcriptional cascade is active in swarmer cells}

There are several arguments pointing to the TacA transcriptional activator as the unidentified PleC- and CtrAdependent transcription factor that binds to $\mathrm{P}_{\text {spmx }}$. First, since the accumulation of the tac $A$ transcript slightly precedes that of $\operatorname{spmX}$ (Laub et al. 2000; McGrath et al. 2007), the temporal pattern of tacA expression is consistent with the proposed role of TacA as transcriptional regulator of $\operatorname{spmX}$. Second, the abundance of the $\operatorname{tac} A$ message is dramatically reduced in the absence of PleC, a feature $\operatorname{tac} A$ shares with pilA and $\operatorname{spmX}$ (Chen et al. 2006). Third, the spm $X$ promoter sequence suggests that an RNAP holoenzyme containing a $\sigma^{54}$ factor $\left(E \sigma^{54}\right)$ transcribes spmX (Fig. 6D; McGrath et al. 2007). E $\sigma^{54}$-catalyzed transcription normally requires a coactivator of a specialized clade of response regulators (Popham et al. 1989|, of which there are only four encoded in the Caulobacter genome and TacA is one of them (Marques et al. 1997). 
Radhakrishnan et al.

Figure 7. The absence of SpmX underlies the Divj localization defect in pleC and ctrA401 mutants. $(A, B)$ Immunoblots of pleC::Tn5 divJ-gfp and ctrA401 divJgfp cells upon expression of TacA or SpmX from a xylose-inducible promoter on a low-copy plasmid $\left(\mathrm{pP}_{\mathrm{xy}} \mathrm{I}^{-}\right.$ tac $A$ ). In $A$, cells were grown in PYE containing tetracycline $(1 \mu \mathrm{g} / \mathrm{mL})$ and $20 \mathrm{mM}$ xylose, except for the strain containing $\mathrm{pP}_{x y}{ }^{-}$spm $X$ that was grown in $2 \mathrm{mM}$ xylose and tetracycline. In $B$, cells were grown in PYE containing tetracycline $(1 \mu \mathrm{g} / \mathrm{mL})$ as well as $20 \mathrm{mM}$ xylose (Xyl) or $20 \mathrm{mM}$ glucose (Glu). (C,D) Fluorescence micrographs showing DivJ-GFP in pleC::Tn5 divJ-gfp $(C)$ and ctrA401 divJ-gfp $(D)$ cells that were grown in PYE containing tetracycline $(1 \mu \mathrm{g} / \mathrm{mL})$ and $20 \mathrm{mM}$ xylose and harbored either plasmid $\left.\mathrm{pP}_{x y}\right]^{-t a c A}$ or the empty vector. $(E)$ Localization of DivJ-GFP in a ple C::Tn5 mutant that expresses SpmX from a xyloseinducible promoter on a low-copy plasmid $\left(\mathrm{pP}_{\mathrm{xyl}}-\mathrm{spmX}\right)$ or with a complementing plasmid harboring the pleC gene. No localization is observed in strains with the empty vector. Cells were grown in PYE containing tetracycline $(1 \mu \mathrm{g} / \mathrm{mL})$. Bars, $\sim 2 \mu \mathrm{m}$.

A

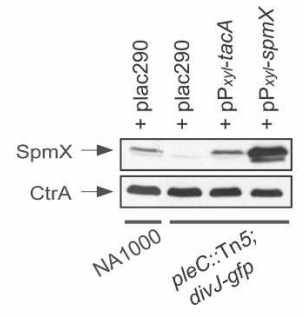

C

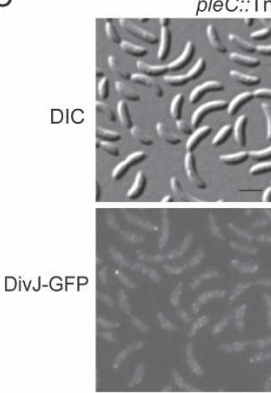

E

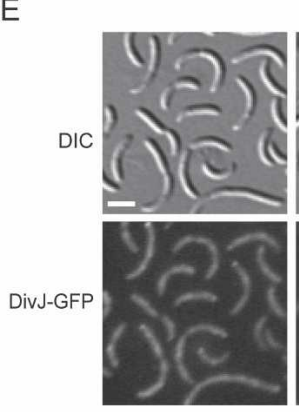

+ pMR20
B

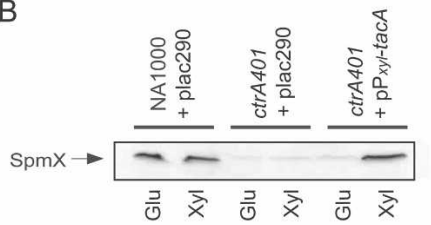

D

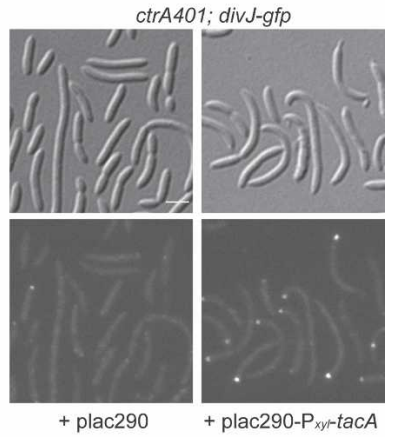

pleC:::Tn5; divJ-gfp

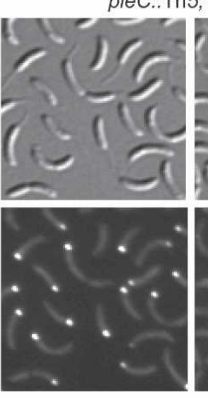

+ pMR20-pleC

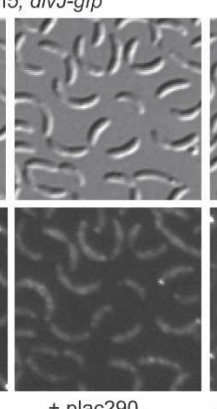

+ plac290

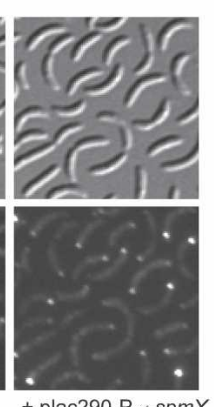

+ plac290-P $x y-\operatorname{spmX}$
Immunoblot analysis and $\mathrm{P}_{\text {spmX }}-1 a c Z$ reporter assays were used to test if TacA regulates $\operatorname{spm} X$ expression. A marked reduction of cellular SpmX levels was observed in strains mutant for TacA or $\sigma^{54}$ (RpoN) (Fig. 6A). Moreover, the $\mathrm{P}_{\text {spmX }}-1 a c Z$ reporter was only $10 \%$ as active in these mutants compared with the wild-type strain (Fig. $6 \mathrm{C})$. Like other response regulators, TacA is also subject to regulation by phosphorylation at a conserved aspartic acid residue (at position 54) (Stock et al. 1990). The ShkA hybrid histidine kinase was shown recently to initiate a phosphorelay that activates TacA by phosphorylation (Biondi et al. 2006b). Consistent with the idea that active TacA stimulates transcription of $\operatorname{spmX}$, disruption of the shk $A$ gene severely impairs SpmX accumulation and $\mathrm{P}_{\text {spmX }}-1$ lac $Z$ activity (Fig. 6A,C). To determine if regulation of spmX transcription by TacA is direct, we conducted qChIP experiments using a polyclonal antibody to TacA. The spmX promoter precipitated specifically and efficiently from lysates of wild-type cells, but not from those of $\triangle$ tacA cells (Fig. 6F; Supplemental Fig. S7B). The staR gene that encodes a regulator of stalk elongation shares promoter sequence motifs with spmX (McGrath et al. 2007) and is thought to be directly regulated by TacA (Biondi et al. 2006b). Our qChIP experiments also demonstrated that TacA interacts efficiently in vivo with the promoter of staR (Fig. 6F), but not with that of two other PleC-dependent genes (CC1695 and CC0167) (Supplemental Fig. S7B). These findings, along with the immunoblots showing that TacA is present in the swarmer cell compartment (Fig. 4A), support the view that TacA binds to $\mathrm{P}_{\text {spmX }}$ in swarmer cells to stimulate transcription by $\mathrm{E \sigma}^{54}$ (Fig. 1B).

Why is $\operatorname{spmX}$ transcription impaired in $\Delta$ pleC cells? Previous experiments implicated that $t a c A$ transcription is under the control of CtrA (Marques et al. 1997), suggesting that $\Delta$ pleC cells might be unable to activate spmX transcription because they lack sufficient amounts of TacA. As expected, immunoblotting revealed that TacA levels are markedly reduced in extracts from $\triangle p l e C$ and ctrA401 cells compared with those from wild-type cells (Fig. 6E). The tacA promoter features a CtrA box that overlaps the -35 promoter motif (Marques et al. 1997), providing further support for the hypothesis that a decrease in CtrA $\sim \mathrm{P}$ is responsible for the reduction of TacA in $\Delta p l e C$ cells. Whether or not CtrA binds the tacA promoter in vivo is unknown. Our qChIP experiments revealed that the $\operatorname{tac} A$ promoter is indeed an in vivo target of CtrA (Fig. 6G). The tacA promoter precipitated with efficiency comparable with the fliL promoter (Supplemental Fig. S7A). Next, we used qChIP analysis to explore if CtrA occupancy at the tacA promoter is compromised in $\Delta p l e C$ cells compared with wild-type 
cells. As shown in Figure 6G, CtrA binding to the tac $A$ promoter is reduced by $60 \%$ when $\mathrm{PleC}$ is absent, thus explaining the reduction of TacA in $\Delta p l e C$ mutant cells. Based on this data, we propose the following sequence of events. First, low CtrA P levels caused by the loss of PleC prevent efficient tacA transcription, which in turn causes a reduction in TacA steady-state levels below the threshold necessary to support spmX transcription. Since $s p m X$ is not transcribed, cells are devoid of SpmX, the localization determinant for DivJ, and, thus, DivJ remains dispersed.

If our interpretation of this transcriptional hierarchy is correct, then SpmX expression should be restored to ple $C$ or $\operatorname{ctr} A$ mutant cells when TacA is expressed from a xylose-inducible $\mathrm{P}_{x y 1}$-tacA construct on a low-copynumber plasmid. In support of our model, Figure 7, A and $\mathrm{B}$, shows that inducing expression of TacA upon administering xylose restored wild-type levels of SpmX to ctrA401 and $\Delta$ pleC mutant cells. Based on these data, we surmise that $s p m X$ transcription in the swarmer cell involves an intricate transcriptional regulatory cascade following the order $\mathrm{PleC} \rightarrow \mathrm{CtrA} \rightarrow \mathrm{TacA} \rightarrow \mathrm{SpmX}$.

\section{SpmX couples PleC signaling to DivJ localization}

Since $\operatorname{ctr} A 401$ cells are devoid of SpmX, they should be unable to direct DivJ to the pole. As expected, only diffuse fluorescence was observed in ctrA401 divJ-gfp cells (Fig. 7D). Since the $\mathrm{P}_{x y}$ l $^{-t a c A}$ construct endows both ctrA401 and pleC mutant cells with SpmX, we asked if this plasmid could remedy the DivJ localization defect of these cells. Indeed, ctrA401 divJ-gfp or pleC::Tn5 divJ$g f p$ cells bearing the $\mathrm{P}_{x y l}$-tacA plasmid sequestered DivJGFP to the pole (Fig. 7C,D) in a xylose-dependent manner. Interestingly, the $\mathrm{P}_{x y} I^{-t a c A}$ plasmid also restored stalk biogenesis to the pleC mutant (Supplemental Fig. S8), indicating that DivJ localization and stalk biogenesis at the G1-S transition rely on TacA whose production is dependent on PleC signaling. To test if SpmX is sufficient to direct DivJ to the pole in the absence of PleC, we expressed SpmX from a $\mathrm{P}_{x y} l^{-}$spmX plasmid in $p l e C:: \operatorname{Tn} 5$ divJ-gfp cells. As expected, $\mathrm{P}_{x y l^{-}}$spmX induced the formation of polar DivJ-GFP clusters (Fig. 7E) in the pleC::Tn5 mutant, but not stalks. Thus, as already suggested by the $\Delta \operatorname{spmX}$ phenotype, SpmX is not the TacAdependent gene that promotes stalk synthesis. Instead, SpmX plays a pivotal role in reprogramming the G1 cell into an S-phase cell by the recruitment of DivJ to the nascent stalked pole at the G1-S transition, an event that is prepared by $\mathrm{PleC}$ through its transcriptional regulation of $\operatorname{spmX}$.

\section{SpmX and DivK define critical nodes of an intricate genetic circuit}

The data presented above outline two branches of a putative autoregulatory circuit in which PleC facilitates SpmX production, whereas SpmX promotes the accumulation of DivK P, which PleC acts against. If these de- pendencies indeed form the basis of a closed genetic circuit, PleC must signal $\operatorname{spm} X$ transcription through DivK. Immunoblotting and $\mathrm{P}_{\text {spmX }}-1 a c Z$ reporter experiments confirmed this prediction, as the divKcs mutation overcame the reduction in cellular SpmX levels and the spmX transcriptional defect of $\Delta p l e C$ cells (Fig. 6B,C). Moreover, qChIP analysis showed that CtrA is again efficiently associated with the tacA promoter in $\Delta p l e C$ divKcs cells (Fig. 6G). Based on these results, we position DivK between PleC and CtrA in the spmX transcriptional cascade (Fig. 1B). The view that dephosphorylated DivK signals the onset of $\operatorname{spm} X$ transcription in the swarmer cell compartment stems from the result that the $\Delta$ divJ mutation, which causes DivK to predominate over DivK $\sim \mathrm{P}$, recapitulates the effects of the divKcs mutation on $\mathrm{P}_{\text {spmx }}$-lac $Z$ activity and SpmX accumulation in $\Delta$ pleC cells (Fig. 6B,C). Thus, high DivK P levels that occur at the G1-S transition or upon inactivating PleC will indirectly shut down $\mathrm{P}_{\text {spmx }}$.

On the basis of these results, we propose the following sequence of events that lead to the accumulation of the spmX transcript in G1 (swarmer) cells. (1) The PleC phosphatase directly and/or indirectly lowers DivK P levels; (2) dephosphorylated DivK promotes to CtrA P accumulation; (3) CtrA P subsequently activates the tacA promoter; and (4) with the synthesis and phosphorylation of TacA, transcription of $\operatorname{spmX}$ is initiated by E $\sigma^{54}$ upon stimulation by TacA. (5) Early at the G1-S transition, either regulated translation or an increase in SpmX stability promotes its accumulation, whereupon it localizes to the developing stalked pole. (6) Polar SpmX then recruits and activates the DivJ kinase. (7) DivK is phosphorylated and (8) indirectly turns off transcription of spmX in nascent stalked cells by down-regulating CtrA P, which (9) eliminates de novo production of TacA, while (10) pre-existing TacA is proteolyzed (Fig. 4A).

\section{Discussion}

The spmX gene can be considered a focal point of regulation of the Caulobacter asymmetric division cycle, since it is intricately regulated at multiple levels both in time and space. Commensurate with its sophisticated regulation, the SpmX protein performs functions that act at the heart of this developmental program. SpmX stimulates DivJ at the G1-S transition and, thus, triggers the surge of DivK P. Moreover, SpmX is a localization factor that directs DivJ and, indirectly, DivK to the pole. Finally, transcription of $s p m X$ in the incipient swarmer cell compartment is activated through DivK as part of a PleC-dependent signal transduction cascade. Based on these data, we propose that SpmX and DivK act as critical nodes of an autoregulatory loop interwoven into the genetic circuitry that implements and sustains Caulobacter asymmetric division. DivK activates the transcriptional branch of this loop to prime swarmer cells for the impending transition into stalked cells, while SpmX that accumulates in stalked cells promotes DivK P production, which shuts off $\operatorname{spmX}$ transcription. 
In the absence of SpmX, DivJ is delocalized and its kinase function is impaired. As a result, DivK is poorly phosphorylated and its ability to execute cell specification is compromised. Our result that the requirement for SpmX to stimulate DivJ activity can be overcome with a $\Delta$ pleC mutation illustrates another nuance of the PleC regulatory pathway that is required to inhibit DivJ (Sommer and Newton 1991; Wheeler and Shapiro 1999). It is conceivable that a negative regulatory activity that is induced by PleC inhibits premature activation of residual DivJ found in swarmer cells. If that is true, we hypothesize that SpmX is required to eliminate this activity at the G1-S transition, thereby relieving repression of DivJ. This hypothesis accounts for the observed requirement of SpmX to stimulate DivJ in wild-type cells and also explains the observation that DivJ activity is unrestrained in $\Delta p l e C$ cells, in which both SpmX and this putative inhibitor are absent. Based on this idea, we posit two concurrent functions of SpmX to exert spatiotemporal control over DivJ. First, by recruiting DivJ to the emerging stalked pole at the G1-S transition, SpmX ensures that the bulk of DivJ partitions with the stalked daughter cell at the next cytokinetic event. Thus, this function of SpmX is central for daughter cell-specific distribution of DivJ. Second, SpmX stimulates DivJ activity, possibly by relieving inhibition of the hypothetical negative regulator outlined above. We cannot exclude an alternative model involving a positive regulator of DivJ that is stimulated by SpmX, but that is hyperactivated by another pathway in pleC mutants where SpmX is absent. Intriguingly, DivK was found recently to act as a positive allosteric regulator of the DivJ kinase (R. Paul and U. Jenal, pers. comm.), raising the possibility that DivK itself might represent the aforementioned hypothetical regulator of DivJ.

In addition to the known battery of effectors, at least one unknown phosphodonor of DivK still exists (Wheeler and Shapiro 1999; Ohta and Newton 2003). The regulatory network converging on DivK bears a remarkable resemblance to that regulating SpoOF, a single domain response regulator that plays an important role in committing Bacillus subtilis cells to the sporulation pathway (Stragier and Losick 1996). SpoOF is subject to regulation by phosphorylation and dephosphorylation by multiple kinases and phosphatases (Jiang et al. 2000a,b; Smits et al. 2007). While it is certainly conceivable that another histidine kinase is the source of DivK P in cells lacking both PleC and DivJ (Wheeler and Shapiro 1999), DivK $\sim$ P might also acquire its phosphate from a highenergy phosphometabolite like acetylphosphate (Wolfe 2005). The regulatory pathways controlling DivK are central for the G1-S transition and likely act in concert with others to complete the underlying developmental changes that remodel a swarmer cell into a replicative stalked cell. These include pathways specified by the master transcriptional regulators GcrA and DnaA as well as the SsrA (tmRNA) quality control system, all of which are active during the G1-S transition and facilitate the onset of DNA replication (Keiler and Shapiro 2003a,b; Holtzendorff et al. 2004; Collier et al. 2006).
A fundamental developmental principle that has found precedence in asymmetric cell division in eukaryotes and in prokaryotes is the targeting of cell fate determinants to specific subcellular positions as exemplified in budding yeast, in Drosophila neuroblasts, and in Caulobacter. The localization of SpmX early during the G1-S transition to recruit Divj to the stalked pole can be compared with the asymmetrically localized Par complex in Drosophila neuroblasts, a complex that includes aPKC along with other proteins that are likely required for its recruitment to the apical membrane (Wirtz-Peitz and Knoblich 2006). As for the Par complex, the current challenge for SpmX is to elucidate how this asymmetric positioning is achieved. SpmX might recognize a structural and/or chemical modification in the polar peptidoglycan layer that attracts it to the old cell pole, a view that is supported by our result that the muramidase domain is necessary and sufficient for the localization of SpmX to the stalked pole. Indeed, the cell division apparatus that directs the biosynthesis of the polar peptidoglycan during cytokinesis has been implicated in the deposition of a spatial mark at the newborn cell pole to cue protein localization (MacAlister et al. 1987; Huitema et al. 2006; Lam et al. 2006). Like SpmX, the localization factor PodJ that recruits the PleC phosphatase to the newborn pole contains a motif thought to interact with peptidoglycan (Viollier et al. 2002b; Hinz et al. 2003). This raises the intriguing possibility that the ability of selected proteins to discriminate between the old and the new cell pole could be based on unique features present in the polar peptidoglycan. If that is true, cellular asymmetry in Caulobacter or other bacteria might be founded on this principle. Even bacteria that appear morphologically symmetrical feature an old and a new cell pole that might promote the formation of unique molecular microdomains that can be exploited for specialized polar functions (Shapiro et al. 2002), predicting that such a localization concept could be widespread among prokaryotes.

Another remarkable aspect unearthed in this work is the complex transcriptional cascade that activates spmX in swarmer cells. It is an essential part of a new genetic circuit in which SpmX promotes the production of DivK P, and DivK P in turn terminates the pathway that activates $\operatorname{spm} X$ transcription at the G1-S transition. This is achieved by halting transcription to prevent de novo synthesis of TacA and degrading pre-existing TacA at the G1-S transition. Later in the cell cycle, once the predivisional cell is compartmentalized and DivK $\sim \mathrm{P}$ levels fall in the swarmer cell chamber, the $\operatorname{spm} X$ transcriptional pathway is once again activated. Launching the pathway for spmX transcription before cell separation has been completed ensures that the daughter swarmer cell is born with the $\operatorname{spmX}$ message in anticipation of the impending transition into stalked cells. The synthesis of a transcript in a compartment long before the function of its translation product is required is a fundamental regulatory strategy that also underlies development of the Drosophila embryo (Micklem 1995). Transcripts like nanos, which codes for a key developmental regu- 
lator, are deposited in the egg by the mother before fertilization has occurred (Wang and Lehmann 1991). Once fertilization occurs, the newly formed embryo is already endowed with maternal-derived messages that can implement developmental changes. Many of these maternal mRNAs are later degraded and replaced with other de novo synthesized embryonic transcripts during the midblastula (also known as the oocyte-zygote) transition (Schier 2007). In a remarkable analogy to the fate of these developmental messages, transcripts like $\operatorname{spmX}$ that are synthesized before the birth of the swarmer cell begin to disappear during the swarmer-tostalked cell transition, the time when the synthesis of stalked cell-specific transcripts commences. Thus, the localization of SpmX as well as the underlying $\operatorname{spmX}$ transcriptional cascade both represent striking manifestations of remarkably similar fundamental developmental mechanisms that operate in both eukaryotes and prokaryotes.

\section{Materials and methods}

\section{Strains and growth conditions}

Caulobacter NA1000 (Evinger and Agabian 1977) and derivatives were grown at $30^{\circ} \mathrm{C}$ in PYE, M2G, or M5G, except divKcs derivatives that were grown at $32^{\circ} \mathrm{C}$. Escherichia coli S17-1 (Simon et al. 1983) and EC100D (Epicentre Technologies) were grown at $37^{\circ} \mathrm{C}$ in LB. Motility assays, swarmer cell isolation, intergeneric conjugations, electroporations, and bacteriophage $\Phi C r-30$-mediated generalized transductions were performed as described (Ely 1991; Viollier and Shapiro 2003; Chen et al. 2005).

Isolation of motility mutants NS190, NS349, NS229, and NS217

From a library of 20,000 transposon mutants that were tested for reduced motility on semisolid PYE $(0.3 \%)$ agar plates, $>400$ mutants were isolated and the transposon insertion site was determined (S. Pritchard D. Matteson, E. Huitema, S.K. Radhakrishnan, and P.H. Viollier, in prep.). To uncover unidentified components of the PleC-DivJ-DivK signaling pathway, we screened all mutants with insertions in uncharacterized genes by TEM for pleiotropic phenotypes resembling those exhibited by divI, divK, or pleC strains, such as abnormalities in cell division and/or polar development (Sommer and Newton 1991). As described in the Results section, NS190 and NS349 shared several properties with the divKcs mutant.

NS190 contains a mariner-derived Himar1 insertion at nucleotide position 6336 of AE005889 delivered by conjugation from E. coli S17-1 harboring pHPV414 (Viollier et al. 2004). In NS349, an EZ-Tn5 transposon (Epicentre Technologies) that had been introduced by electroporation was inserted at nucleotide position 5805 of AE005889. Strain NS229 has a HyperMu (Epicentre Technologies) insertion in rpoN (nucleotide position 4011 of AE006018). An EZ-Tn5 insertion at nucleotide 3762 of AE005688 disrupted $\operatorname{shk} A$ in strain NS217.

\section{$\beta$-Galactosidase assays and immunoblots}

$\beta$-Galactosidase assays were performed at $30^{\circ} \mathrm{C}$ as described earlier (Viollier and Shapiro 2003; Huitema et al. 2006). For the production of anti-SpmX and anti-TacA antibodies, the sequences coding for residues $120-351$ of SpmX and 226-488 of TacA were overexpressed in E. coli Rosetta (DE3)/pLysS using
pET28a (Novagen) as $\mathrm{N}$-terminal His ${ }_{6}$-tagged variants and were purified using Ni-NTA agarose (Qiagen). Purified proteins were excised from a $12.5 \%$ SDS polyacrylamide gel and used to immunize rabbits (Josman LLC). Antisera were used at the following dilutions: anti-SpmX $(1: 50,000)$, anti-TacA $(1: 30,000)$, antiMcpA $(1: 10 ; 000)$, anti-CtrA $(1: 10,000)$, anti-DivJ $(1: 10,000)$, antimRFP1 (1:5000), anti-DivK (1:10,000), and anti-PilA (1:5000) (Alley et al. 1992; Domian et al. 1997; Wheeler and Shapiro 1999; Jacobs et al. 2001; Viollier et al. 2002a; Chen et al. 2005).

\section{Microscopy}

TEM was performed as described earlier (Skerker and Shapiro 2000; Huitema et al. 2006). For fluorescence and DIC imaging, a Nikon Eclipse 90i microscope fitted with a 100× oil TIRF (1.45 numerical aperture) objective and a CoolSnap $\mathrm{HQ}^{2}$ camera (Photometrics) with suitable filters was used. Images were acquired and processed using MetaMorph version 7.0 (Molecular Devices). IFM was conducted as described (Viollier et al. 2002a) using the anti-SpmX antiserum at a dilution of 1:2000.

\section{In vivo ${ }^{32} \mathrm{P}$ labeling}

In vivo phosphorylation experiments were performed with certain modifications of a previously described method (Domian et al. 1997). A single colony of cells picked from a PYE agarose plate was washed with M5G medium lacking phosphate and was grown overnight in M5G with $0.05 \mathrm{mM}$ phosphate to an optical density of 0.3 at $660 \mathrm{~nm}$. One milliliter of culture was labeled for $4 \mathrm{~min}$ at $28^{\circ} \mathrm{C}$ using $30 \mu \mathrm{Ci}$ of $\gamma-\left[{ }^{32} \mathrm{P}\right] \mathrm{ATP}$. Following lysis, proteins were immunoprecipitated with $3 \mu \mathrm{L}$ of anti-DivJ or anti-DivK antiserum. The precipitates were resolved by SDSPAGE, and $\left[{ }^{32} \mathrm{P}\right]$-labeled DivK or DivJ were quantified using a Storm 820 PhosphorImager and ImageQuant software version 4.0 (Molecular Dynamics) and were normalized to the relative cellular content as determined by immunoblotting of lysates.

\section{Release of periplasmic proteins}

A previously described procedure for releasing periplasmic protein upon spheroblasting was used (Judd et al. 2005) with minor modifications. Ready-Lyse lysozyme solution (Epicentre) was used at a concentration of $9 \mathrm{U} / \mu \mathrm{L}$, and the cells were incubated for $10 \mathrm{~min}$ at room temperature. Spheroblasts were centrifuged at $4500 \mathrm{~g}$ for $5 \mathrm{~min}$, and samples were collected and then subjected to immunoblot analysis.

\section{Sequence analysis}

The DAS (http://www.sbc.su.se/ miklos/DAS/maindas.html) server was used for the prediction of transmembrane domains in SpmX.

Immunoprecipitations, strain, and plasmid constructions Details for these procedures can be found in the Supplemental Material.

\section{Acknowledgments}

We thank Sean Pritchard for excellent technical assistance; Piet de Boer and Arne Rietsch for critical reading of the manuscript; and Mike Laub, Christine Jacobs-Wagner, and Kathleen Ryan for materials. Supported by funds from the School of Medicine, the Mount Sinai Health Care Foundation, and the U.S. Depart- 
ment of Energy Office of Science (BER, grant no. DE-FG0205ER64136) to P.V., and start-up funds from the Max Planck Society to M.T.

\section{References}

Alley, M.R., Maddock, J.R., and Shapiro, L. 1992. Polar localization of a bacterial chemoreceptor. Genes \& Dev. 6: 825-836.

Biondi, E.G., Reisinger, S.J., Skerker, J.M., Arif, M., Perchuk, B.S., Ryan, K.R., and Laub, M.T. 2006a. Regulation of the bacterial cell cycle by an integrated genetic circuit. Nature 444: 899-904.

Biondi, E.G., Skerker, J.M., Arif, M., Prasol, M.S., Perchuk, B.S., and Laub, M.T. 2006b. A phosphorelay system controls stalk biogenesis during cell cycle progression in Caulobacter crescentus. Mol. Microbiol. 59: 386-401.

Chen, J.C., Viollier, P.H., and Shapiro, L. 2005. A membrane metalloprotease participates in the sequential degradation of a Caulobacter polarity determinant. Mol. Microbiol. 55: 1085-1103.

Chen, J.C., Hottes, A.K., McAdams, H.H., McGrath, P.T., Viollier, P.H., and Shapiro, L. 2006. Cytokinesis signals truncation of the PodJ polarity factor by a cell cycle-regulated protease. $E M B O$ J. 25: 377-386.

Collier, J., Murray, S.R., and Shapiro, L. 2006. DnaA couples DNA replication and the expression of two cell cycle master regulators. $E M B O J$. 25: 346-356.

Domian, I.J., Quon, K.C., and Shapiro, L. 1997. Cell type-specific phosphorylation and proteolysis of a transcriptional regulator controls the G1-to-S transition in a bacterial cell cycle. Cell 90: 415-424.

Ely, B. 1991. Genetics of Caulobacter crescentus. Methods Enzymol. 204: 372-384.

Evinger, M. and Agabian, N. 1977. Envelope-associated nucleoid from Caulobacter crescentus stalked and swarmer cells. J. Bacteriol. 132: 294-301.

Hinz, A.J., Larson, D.E., Smith, C.S., and Brun, Y.V. 2003. The Caulobacter crescentus polar organelle development protein PodJ is differentially localized and is required for polar targeting of the PleC development regulator. Mol. Microbiol. 47: 929-941.

Holtje, J.V. 1996. Bacterial lysozymes. EXS 75: 65-74.

Holtzendorff, J., Hung, D., Brende, P., Reisenauer, A., Viollier, P.H., McAdams, H.H., and Shapiro, L. 2004. Oscillating global regulators control the genetic circuit driving a bacterial cell cycle. Science 304: 983-987.

Horvitz, H.R. and Herskowitz, I. 1992. Mechanisms of asymmetric cell division: Two Bs or not two Bs, that is the question. Cell 68: 237-255.

Huitema, E., Pritchard, S., Matteson, D., Radhakrishnan, S.K., and Viollier, P.H. 2006. Bacterial birth scar proteins mark future flagellum assembly site. Cell 124: 1025-1037.

Hung, D.Y. and Shapiro, L. 2002. A signal transduction protein cues proteolytic events critical to Caulobacter cell cycle progression. Proc. Natl. Acad. Sci. 99: 13160-13165.

Iniesta, A.A., McGrath, P.T., Reisenauer, A., McAdams, H.H., and Shapiro, L. 2006. A phospho-signaling pathway controls the localization and activity of a protease complex critical for bacterial cell cycle progression. Proc. Natl. Acad. Sci. 103: 10935-10940.

Jacobs, C., Hung, D., and Shapiro, L. 2001. Dynamic localization of a cytoplasmic signal transduction response regulator controls morphogenesis during the Caulobacter cell cycle. Proc. Nat1. Acad. Sci. 98: 4095-4100.

Jiang, M., Grau, R., and Perego, M. 2000a. Differential process- ing of propeptide inhibitors of Rap phosphatases in Bacillus subtilis. J. Bacteriol. 182: 303-310.

Jiang, M., Shao, W., Perego, M., and Hoch, J.A. 2000b. Multiple histidine kinases regulate entry into stationary phase and sporulation in Bacillus subtilis. Mol. Microbiol. 38: 535-542.

Judd, E.M., Comolli, L.R., Chen, J.C., Downing, K.H., Moerner, W.E., and McAdams, H.H. 2005. Distinct constrictive processes, separated in time and space, divide Caulobacter inner and outer membranes. J. Bacteriol. 187: 6874-6882.

Keiler, K.C. and Shapiro, L. 2003a. tmRNA in Caulobacter crescentus is cell cycle regulated by temporally controlled transcription and RNA degradation. J. Bacteriol. 185: 1825-1830.

Keiler, K.C. and Shapiro, L. 2003b. tmRNA is required for correct timing of DNA replication in Caulobacter crescentus. J. Bacteriol. 185: 573-580.

Lam, H., Matroule, J.Y., and Jacobs-Wagner, C. 2003. The asymmetric spatial distribution of bacterial signal transduction proteins coordinates cell cycle events. Dev. Cell 5: 149-159.

Lam, H., Schofield, W.B., and Jacobs-Wagner, C. 2006. A landmark protein essential for establishing and perpetuating the polarity of a bacterial cell. Cell 124: 1011-1023.

Laub, M.T., McAdams, H.H., Feldblyum, T., Fraser, C.M., and Shapiro, L. 2000. Global analysis of the genetic network controlling a bacterial cell cycle. Science 290: 2144-2148.

Laub, M.T., Chen, S.L., Shapiro, L., and McAdams, H.H. 2002. Genes directly controlled by CtrA, a master regulator of the Caulobacter cell cycle. Proc. Natl. Acad. Sci. 99: 4632-4637.

MacAlister, T.J., Cook, W.R., Weigand, R., and Rothfield, L.I. 1987. Membrane-murein attachment at the leading edge of the division septum: A second membrane-murein structure associated with morphogenesis of the Gram-negative bacterial division septum. J. Bacteriol. 169: 3945-3951.

Marques, M.V., Gomes, S.L., and Gober, J.W. 1997. A gene coding for a putative $\sigma^{54}$ activator is developmentally regulated in Caulobacter crescentus. J. Bacteriol. 179: 5502-5510.

Matroule, J.Y., Lam, H., Burnette, D.T., and Jacobs-Wagner, C. 2004. Cytokinesis monitoring during development: Rapid pole-to-pole shuttling of a signaling protein by localized kinase and phosphatase in Caulobacter. Cell 118: 579-590.

McGrath, P.T., Lee, H., Zhang, L., Iniesta, A.A., Hottes, A.K., Tan, M.H., Hillson, N.J., Hu, P., Shapiro, L., and McAdams, H.H. 2007. High-throughput identification of transcription start sites, conserved promoter motifs and predicted regulons. Nat. Biotechnol. 25: 584-592.

Micklem, D.R. 1995. mRNA localisation during development. Dev. Biol. 172: 377-395.

Ohta, N. and Newton, A. 2003. The core dimerization domains of histidine kinases contain recognition specificity for the cognate response regulator. J. Bacteriol. 185: 4424-4431.

Ohta, N., Lane, T., Ninfa, E.G., Sommer, J.M., and Newton, A. 1992. A histidine protein kinase homologue required for regulation of bacterial cell division and differentiation. Proc. Natl. Acad. Sci. 89: 10297-10301.

Pierce, D.L., O'Donnol, D.S., Allen, R.C., Javens, J.W., Quardokus, E.M., and Brun, Y.V. 2006. Mutations in DivL and CckA rescue a divJ null mutant of Caulobacter crescentus by reducing the activity of CtrA. J. Bacteriol. 188: 2473-2482.

Popham, D.L., Szeto, D., Keener, J., and Kustu, S. 1989. Function of a bacterial activator protein that binds to transcriptional enhancers. Science 243: 629-635.

Quon, K.C., Marczynski, G.T., and Shapiro, L. 1996. Cell cycle control by an essential bacterial two-component signal transduction protein. Cell 84: 83-93.

Quon, K.C., Yang, B., Domian, I.J., Shapiro, L., and Marczynski, G.T. 1998. Negative control of bacterial DNA replication by a cell cycle regulatory protein that binds at the chromosome 
origin. Proc. Nat1. Acad. Sci. 95: 120-125.

Schier, A.F. 2007. The maternal-zygotic transition: Death and birth of RNAs. Science 316: 406-407.

Shapiro, L. and Losick, R. 1997. Protein localization and cell fate in bacteria. Science 276: 712-718.

Shapiro, L., McAdams, H.H., and Losick, R. 2002. Generating and exploiting polarity in bacteria. Science 298: 1942-1946.

Simon, R., Priefer, U., and Puhler, A. 1983. A broad host range mobilization system for in vivo genetic engineering: Transposon mutagenesis in Gram negative bacteria. Biotechnology (N.Y.) 1: 784-790.

Skerker, J.M. and Laub, M.T. 2004. Cell-cycle progression and the generation of asymmetry in Caulobacter crescentus. Nat. Rev. Microbiol. 2: 325-337.

Skerker, J.M. and Shapiro, L. 2000. Identification and cell cycle control of a novel pilus system in Caulobacter crescentus. EMBO T. 19: 3223-3234.

Smits, W.K., Bongiorni, C., Veening, J.W., Hamoen, L.W., Kuipers, O.P., and Perego, M. 2007. Temporal separation of distinct differentiation pathways by a dual specificity RapPhr system in Bacillus subtilis. Mol. Microbiol. 65: 103-120.

Sommer, J.M. and Newton, A. 1991. Pseudoreversion analysis indicates a direct role of cell division genes in polar morphogenesis and differentiation in Caulobacter crescentus. Genetics 129: 623-630.

Stock, J.B., Stock, A.M., and Mottonen, J.M. 1990. Signal transduction in bacteria. Nature 344: 395-400.

Stragier, P. and Losick, R. 1996. Molecular genetics of sporulation in Bacillus subtilis. Annu. Rev. Genet. 30: 297-341.

Thanbichler, M., Iniesta, A.A., and Shapiro, L. 2007. A comprehensive set of plasmids for vanillate- and xylose-inducible gene expression in Caulobacter crescentus. Nucleic Acids Res. 35: e137. doi: 10.1093/nar/gkm818.

Viollier, P.H. and Shapiro, L. 2003. A lytic transglycosylase homologue, PleA, is required for the assembly of pili and the flagellum at the Caulobacter crescentus cell pole. Mol. Microbiol. 49: 331-345.

Viollier, P.H., Sternheim, N., and Shapiro, L. 2002a. A dynamically localized histidine kinase controls the asymmetric distribution of polar pili proteins. EMBO J. 21: 4420-4428.

Viollier, P.H., Sternheim, N., and Shapiro, L. 2002b. Identification of a localization factor for the polar positioning of bacterial structural and regulatory proteins. Proc. Nat1. Acad. Sci. 99: 13831-13836.

Viollier, P.H., Thanbichler, M., McGrath, P.T., West, L., Meewan, M., McAdams, H.H., and Shapiro, L. 2004. Rapid and sequential movement of individual chromosomal loci to specific subcellular locations during bacterial DNA replication. Proc. Natl. Acad. Sci. 101: 9257-9262.

Wang, C. and Lehmann, R. 1991. Nanos is the localized posterior determinant in Drosophila. Cell 66: 637-647.

Wang, S.P., Sharma, P.L., Schoenlein, P.V., and Ely, B. 1993. A histidine protein kinase is involved in polar organelle development in Caulobacter crescentus. Proc. Nat1. Acad. Sci. 90: 630-634.

Wheeler, R.T. and Shapiro, L. 1999. Differential localization of two histidine kinases controlling bacterial cell differentiation. Mol. Cell 4: 683-694.

Wirtz-Peitz, F. and Knoblich, J.A. 2006. Lethal giant larvae take on a life of their own. Trends Cell Biol. 16: 234-241.

Wolfe, A.J. 2005. The acetate switch. Microbiol. Mol. Biol. Rev. 69: $12-50$.

Wu, J., Ohta, N., and Newton, A. 1998. An essential, multicomponent signal transduction pathway required for cell cycle regulation in Caulobacter. Proc. Natl. Acad. Sci. 95: 14431448. 


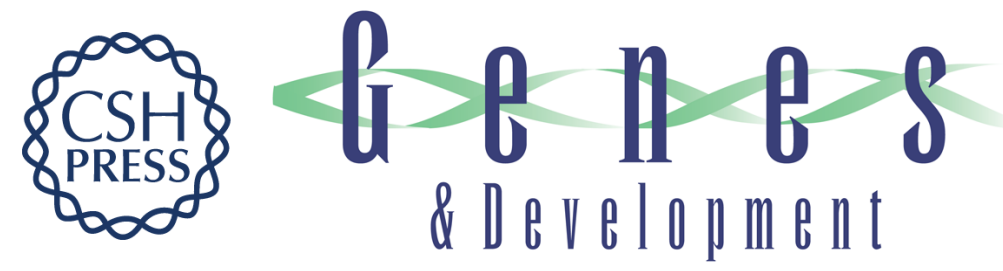

\section{The dynamic interplay between a cell fate determinant and a lysozyme homolog drives the asymmetric division cycle of Caulobacter crescentus}

Sunish Kumar Radhakrishnan, Martin Thanbichler and Patrick H. Viollier

Genes Dev. 2008, 22:

Access the most recent version at doi:10.1101/gad.1601808

Supplemental http://genesdev.cshlp.org/content/suppl/2008/01/16/22.2.212.DC1

Material

References This article cites 58 articles, 30 of which can be accessed free at: http://genesdev.cshlp.org/content/22/2/212.full.html\#ref-list-1

License

Email Alerting Receive free email alerts when new articles cite this article - sign up in the box at the top Service right corner of the article or click here.

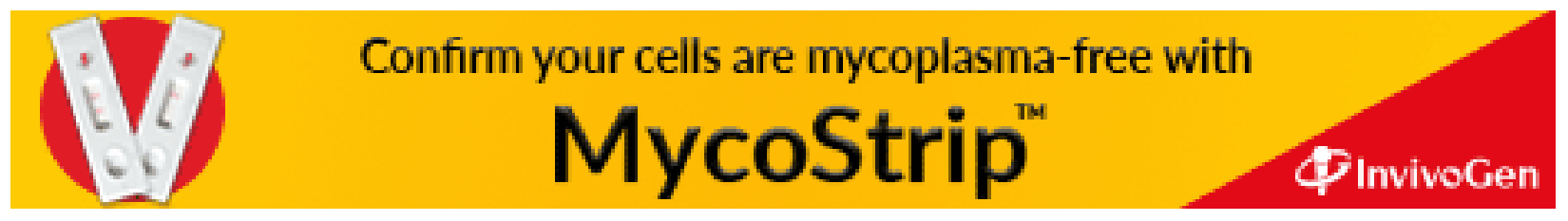

